\title{
A Novel Method for Quantifying Intestinal Inflammatory Burden in Inflammatory Bowel Disease Using Register Data
}

This article was published in the following Dove Press journal: Clinical Epidemiology

\author{
Jordan E Axelrad (1D) ${ }^{1, *}$ \\ Michael C Sachs $\mathbb{D}^{2, *}$ \\ Jonas F Ludvigsson (iD ${ }^{2-5}$ \\ Ola Olén $\mathbb{D}^{6-8}$
}

On behalf of The SWIBREG Study Group

'Division of Gastroenterology, Department of Medicine, NYU School of Medicine, New York, New York, USA; ${ }^{2}$ Department of Medical Epidemiology and Biostatistics, Karolinska Institutet, Stockholm, Sweden; ${ }^{3}$ Department of Pediatrics, Orebro University Hospital, Orebro, Sweden; ${ }^{4}$ Division of Epidemiology and Public Health, School of Medicine, University of Nottingham, Nottingham, UK; ${ }^{5}$ Department of Medicine, Columbia University College of Physicians and Surgeons, New York, New York, USA; ${ }^{6}$ Clinical Epidemiology Division, Department of Medicine Solna, Karolinska Institutet, Stockholm, Sweden; ${ }^{7}$ Sachs' Children and Youth Hospital, Stockholm South General Hospital, Stockholm, Sweden; ${ }^{8}$ Department of Clinical Science and Education Södersjukhuset, Karolinska Institutet, Stockholm, Sweden

*These authors contributed equally to this work
Background: The Swedish Quality Register for Inflammatory Bowel Disease (SWIBREG) contains clinical data for the study of inflammatory bowel disease (IBD). The Epidemiology Strengthened by histoPathology Reports in Sweden (ESPRESSO) cohort was recently established for the study of gastrointestinal histopathology. We aimed to develop and validate a histology score from ESPRESSO using clinical information from SWIBREG, and secondarily, to evaluate the association of the score on IBD-related hospitalization.

Methods: In a nationwide, population-based cohort study of patients with IBD during 1969-2017, we linked endoscopic inflammation in SWIBREG with histologic inflammation in ESPRESSO. We established a clinically interpretable model for predicting the endoscopic score from histology using scalable Bayesian rule lists to define a SNOMED-based histology score applicable to the ESPRESSO cohort. We also assessed the impact of baseline endoscopic and histology scores on time to IBD-related hospitalization.

Results: We identified 5225 individuals with IBD comprising 11,051 endoscopic assessments in SWIBREG linked to a histopathology record in ESPRESSO. We created predictive models to calculate a SNOMED-based histology score which predicted the endoscopic score. Split-sample validated areas under the ROC curves for the score predicting a non-zero endoscopic score were $0.80(0.78-0.81)$ in UC, $0.70(0.68-0.72)$ in $\mathrm{CD}$, and $0.76(0.73-0.78)$ in IBD-U. In a subset of 2741 individuals with an initial IBD diagnosis and a corresponding record in ESPRESSO with an endoscopic assessment in SWIBREG, the baseline endoscopic and histology scores were associated with time to IBD-related hospitalization (endoscopy log-rank UC $\mathrm{p}<0.001, \mathrm{CD} \mathrm{p}=0.020$, IBD-U $\mathrm{p}<0.001$; histology log-rank UC $\mathrm{p}=0.018, \mathrm{CD}$ $\mathrm{p}=0.960$, IBD-U $\mathrm{p}=0.034$ ).

Conclusion: Histopathology data in ESPRESSO accurately predict endoscopic scores in SWIBREG. Baseline endoscopic and histologic scores were associated with time to IBDrelated hospitalization, particularly in UC. The SNOMED-based histology score can be used as a measure of disease activity in future register-based IBD studies.

Keywords: Crohn's disease, ulcerative colitis, endoscopic healing, mucosal healing, histologic healing

\section{Introduction}

Inflammatory bowel diseases (IBD), comprising Crohn's disease (CD) and ulcerative colitis (UC), are chronic gastrointestinal conditions. IBD is thought to be driven by inappropriate immune responses to an altered gut microbiome, or dysbiosis, with a disease course characterized by remitting and relapsing episodes of
Correspondence: Jordan E Axelrad Department of Medicine, NYU School of Medicine, 240 East 38th Street, New York, NY, 10016, USA

Email jordan.axelrad@nyulangone.org 
inflammation, or flares. ${ }^{1,2}$ Duration and severity of intestinal inflammation are linked with complications of disease including colorectal cancer or requiring surgery. ${ }^{3}$ Recently, not only clinical remission but also endoscopic healing $(\mathrm{EH})$, defined as the absence of ulcerations and erosions on endoscopy, has emerged as an important treatment goal in patients with IBD. ${ }^{4,5} \mathrm{EH}$ is associated with improved outcomes, including higher rates of clinical remission, corticosteroid-free clinical remission, avoidance of intestinal surgery, decreased hospitalization, and improved quality of life. ${ }^{6-10}$ Despite the advantages of an endoscopic evaluation in the objective assessment of remission, $\mathrm{EH}$ is also not always an accurate indicator of histologic activity as microscopic evidence of inflammation is present even in patients with clinically and endoscopically quiescent disease. $^{11-15}$ Several studies have shown that histologic healing $(\mathrm{HH})$ is associated with favorable clinical outcomes beyond $\mathrm{EH}$ and is a negative predictor of disease relapse and of colorectal neoplasia risk. $7,12,13,16-18,20,24$ However, previous studies of mucosal healing, defined as the presence of $\mathrm{EH}$ and $\mathrm{HH}$, in IBD comprise poorly generalizable trial or observational data from tertiary centers with limited follow up. Moreover, previous studies correlating endoscopic and histological disease activity are limited. $^{14,25}$

In Sweden, publicly funded healthcare is universally accessible to all residents with interactions recorded in government-administered national registers (healthcare utilization, pharmacy, outpatient care, inpatient care, cancer, mortality, socio-economic demographics, and immigration/emigration status) with nearly complete coverage. Sweden is therefore an ideal setting for high quality prognostic and safety studies in IBD. ${ }^{26,27}$ Despite the importance of both clinical activity and histopathology data in routine IBD care, none of the Swedish governmentadministered national healthcare registers contain direct measures of clinical, macroscopic, or histological disease activity in IBD. ${ }^{28}$

The Swedish Quality Register for IBD (SWIBREG, governed by the Swedish Societies of Gastroenterology, Pediatric Gastroenterology, Colorectal Surgery, and Gastroenterological Nursing), established in 2005, contains detailed clinical IBD data, including disease characteristics and endoscopic measures of disease activity. ${ }^{29}$ SWIBREG has a national coverage of more than $60 \%$, with 50,780 registered patients as of March 2020, but only a minority have complete, longitudinal information regarding endoscopic activity required for the assessment of EH. The ESPRESSO (Epidemiology Strengthened by histoPathology Reports in Sweden) study, established in 2017, comprises histopathology data between 1965 and 2017 coded by SNOMED from all 28 pathology departments in Sweden from the gastrointestinal tract (pharynx to anus) and has high coverage since the mid-1990s. ${ }^{30}$ ESPRESSO contains 6.1 million gastrointestinal biopsies from 2.1 million unique individuals, and has been validated for the study of Celiac disease, ${ }^{31,32}$ serrated polyps, ${ }^{33}$ and microscopic colitis. ${ }^{34}$

To what extent histopathology data in ESPRESSO can predict EH as captured in SWIBREG has not been established. Reliable measures of mucosal healing, including $\mathrm{EH}$ and $\mathrm{HH}$, are critical for the evaluation of IBD prognosis and drug safety as a function of degree and/or duration of inflammation in future register-based studies. Furthermore, since $\mathrm{EH}$ and $\mathrm{HH}$ do not always concur, it is important to measure their individual associations with IBD outcomes that reflect disease activity, such as IBDrelated hospitalization.

We therefore aimed to develop and validate a score for measuring intestinal histologic inflammation based on SNOMED codes in ESPRESSO using endoscopic measures of intestinal inflammation from SWIBREG as the reference standard. We subsequently aimed to clinically validate the SNOMED-based histology score by assessing its association with IBD-related hospitalization.

\section{Methods}

\section{Register Sources}

International classification of disease (ICD) codes are captured prospectively in routine medical practice in the Swedish National Patient Registers (NPR). Data registered in the NPR include main diagnosis, secondary diagnoses, external cause of injury and poisoning, procedure codes (nationwide coverage for both inpatient and hospital-based outpatient care since 2001), ${ }^{35}$ and personal identity number to allow for linkage with other Swedish registers. ${ }^{36}$ SWIBREG contains clinical IBD data that are either missing in the NPR or lacking in detail, including disease duration, extent, surgery, prescribed and administered drugs as well as their side-effects, disease activity and complications, and laboratory data. ${ }^{29,37,38}$ While SWIBREG was established in 2005, it contains information on patients diagnosed as early as 1996 through chart review. The ESPRESSO study, comprising gastrointestinal 
histopathology coded by location (topography) and histologic appearance (morphology) was also used. ${ }^{30}$

\section{Study Population}

All patients with $\geq 1$ ICD code for IBD (CD, UC, or IBDUnclassified (IBD-U)) in the NPR and $\geq 1$ colorectal biopsy suggestive of IBD by systematized Nomenclature of Medicine (SNOMED) codes in ESPRESSO were identified. These individuals were linked to SWIBREG data (Supplemental Table 1) by the unique personal identity number (PIN) issued to all Swedish residents. Having $\geq 1$ ICD code for IBD plus a relevant biopsy code has a positive predictive value of $95 \%$ for IBD (personal communication, JF Ludvigsson). We differentiated between IBD subtypes (CD, UC, or IBD-U) as defined by the first diagnostic code in combination with the pathologic criteria according to an established criteria. ${ }^{39}$

\section{Variables}

Data from the NPR included IBD diagnostic listings from outpatient visits and hospitalizations and endoscopic procedures (Supplemental tables 1 and 2). Data from SWIBREG included location of endoscopic assessment (using text descriptions of location; Supplemental Table 2), and endoscopic scoring (Mayo score for $\mathrm{UC}^{40}$ and SWIBREG-CD score for CD). ${ }^{21,29}$ Data from ESPRESSO included morphology (Morphology codes; Supplemental Table 3) and topography (6-digit topography codes; Supplemental Table 2). Morphology codes ranged from normal to more severe abnormalities. Topography codes were used to confirm endoscopic location of biopsies.

\section{Definition of Inflammation and Location in SWIBREG and ESPRESSO}

Degree of inflammation was defined in SWIBREG and ESPRESSO. In SWIBREG, macroscopic endoscopic inflammation was assessed using the Mayo endoscopic subscore (MES) for UC and IBD-U (0-3) and SWIBREGCD score (SCS) for CD (0-3); Supplemental Table 4). In ESPRESSO, microscopic histologic inflammation was assessed using $\mathrm{M}$ codes.

In the SWIBREG database, we collected information on the macroscopic location and extent for each available subject and date when subjects were endoscopically assessed. The text descriptions of the macroscopic location of the assessment were translated into the topographic codes. For each of the topographic location codes T65 (jejunum and ileum), T67 (cecum/ascending colon, transverse colon, and descending/sigmoid colon), and T68 (rectum), we recorded the maximum MES/SCS (0-3) and the specific location where the maximum was observed. Thus, for each subject in SWIBREG, for each date at which they were assessed, and for each of the locations, we had a record of the maximum severity of inflammation. These were considered the primary units of analysis for the score development.

Through the ESPRESSO study, we had records of the date, topography, and morphology of the histology assessment. For each subject, date, and 3-digit topography code (T65, T67, and T68), there could be multiple morphology codes.

\section{Development of SNOMED-Based Histology Score (SWIBREG-ESPRESSO- NPR Linkage)}

We linked ESPRESSO to SWIBREG data (Supplemental

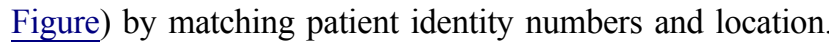
First, we assessed the timing between the SWIBREG assessment and the ESPRESSO record. We considered a match to be finding a histologic ESPRESSO record within 31 days after the endoscopic SWIBREG assessment for the corresponding 3-digit topography code. Next, we randomly split these training data into training (two-thirds) and validation samples (one-third).

In the training sample, we estimated regression models with the MES/SCS as the outcomes and the linked ESPRESSO variables representing the presence of distinct pathology codes as the predictors. We restricted the variables to pathology codes with more than $0.1 \%$ frequency in the sample. Due to the number of unique codes in ESPRESSO and the need for interpretable models, we used the Scalable Bayesian Rule Lists Model to identify Bayesian rule lists that are interpretable with high predictive accuracy (Bayes). ${ }^{41}$ For comparison of predictive performance, we also fit L1 penalized multinomial models for regularization and variable selection, known as the least absolute shrinkage and selection operator (Lasso). ${ }^{42}$ The predicted values from these models represent continuous SNOMED-based histology scores correlated with macroscopic inflammation. In addition to the data driven development of histology scores described above, we also defined a histology severity score based solely on 
clinicians' (JEA, OO, JFL) interpretation (and grouping) of the Swedish pathology codes (Supplemental Table 3).

Development and validation of the prediction models were done separately for $\mathrm{UC}, \mathrm{CD}$ and IBD-U. In the validation sample, we calculated the aforementioned SNOMED-based histology scores using the models that were estimated using the training sample. We then constructed boxplots and receiver operating characteristic (ROC) curves in order to visually assess the correspondence between the different histology scores (referred to as Bayes, Lasso, and Clinical) from ESPRESSO and the endoscopic score from SWIBREG. Differences in the distributions of histology scores by endoscopy score groups were tested using the Kruskal-Wallis test. We also computed the areas under the ROC curves (AUC) with bootstrap 95\% confidence intervals and Cohen's kappa to numerically quantify the agreement between the categorical endoscopy score predicted by SNOMED-based histology scores and the actual endoscopy score. Differences in the AUCs for the different models were tested using a permutation test.

\section{IBD-Related Hospitalization (SWIBREG-ESPRESSO-NPR and ESPRESSO-NPR Linkage)}

After developing and validating the SNOMED-based histology scores, we assessed the impacts of the initial SWIBREG endoscopic score and the ESPRESSO histologic score at IBD diagnosis on IBD-related hospitalization (Supplemental Figure). For this purpose, we restricted to subjects with a recorded endoscopic assessment within 1 year before or after the date of their initial IBD diagnosis. We defined the exposure variables as the baseline SWIBREG endoscopic score and ESPRESSO SNOMEDbased histology score which was closest in time to the initial IBD diagnosis, as long as it was within 1 year. For each of the location codes available on that date, the algorithm was applied to compute a score for each location, and the maximum of the scores over the locations was taken to define the maximum extent of inflammation. The outcome variable is defined as the time to first IBDrelated hospitalization, operationalized as the date of an inpatient visit in the NPR with IBD as the primary diagnosis (ICD 10 code K51 or K50), or end of follow up, not counting IBD hospitalizations during the first 30 days following IBD onset. We linked this sample to the subset of patients available in SWIBREG with a recorded endoscopic score within one year of an IBD diagnosis and then computed SNOMED-based histology scores according to our algorithms so that we could compare the independent and adjusted associations of endoscopic score and our histology score with IBD hospitalization. Kaplan-Meier estimates of the IBD-hospitalization free survival were computed in groups defined by the scores and compared using the Log rank test, and Cox regression was used to estimate adjusted hazard ratios (aHRs) for the impact of baseline nonzero endoscopic score and baseline SNOMED-based histology score on time-to-IBDhospitalization.

Analyses were conducted using $\mathrm{R}$ statistical software (version 3.6.1, R Foundation for Statistical Computing, Vienna, Austria) with the "sblr" (Scalable Bayesian Rule Lists Model. R package version 1.2. https://CRAN. R-project.org/package $=$ sbrl $),{ }^{43}$ plotROC,${ }^{44}$ and survival packages $^{45}$ (R package version 3.1-12, https://CRAN. $\underline{\mathrm{R} \text {-project.org/package=survival)} \text {. }}$.

\section{Ethics}

Data accessed from Swedish patient registers is not freely available. Review and approval were required for this research by an ethics committee. The regional ethics committee in Stockholm approved the study (DNR 2007/785$31 / 5$; 2011/1509-32; 2012/601-32; 2015/0004-31; 2015/ $615-32 ; 2015 / 1030-32)$.

\section{Results}

\section{Study Cohorts}

We identified 5225 individuals with IBD diagnoses in the NPR comprising 11,051 endoscopic assessments in SWIBREG linked to a histopathology record in ESPRESSO to calculate a SNOMED-based histology score (SWIBREG-ESPRESSO-NPR linkage; Table 1, first column; Supplemental Figure). From these individuals, 3448 and 1777 were randomly selected for the training sample and validation subsample, respectively. Separately, we identified 48,449 individuals with initial IBD diagnoses in the NPR since 2002 with a histopathology record in ESPRESSO within 1 year of their IBD diagnosis (ESPRESSO-NPR linkage; Table 1, second column; Supplemental Figure).

Focusing on timing of registration in SWIBREG and a histopathology specimen in ESPRESSO, the majority of histology assessments occurred within 31 days of the SWIBREG endoscopy assessment, largely within 5 days 
Table I Description of Registry Linkage

\begin{tabular}{|c|c|c|}
\hline & $\begin{array}{l}\text { Linkage of SWIBREG- } \\
\text { ESPRESSO-NPR, } \\
\text { n (\%) }\end{array}$ & $\begin{array}{l}\text { Linkage of } \\
\text { ESPRESSO- } \\
\text { NPR, n (\%) }\end{array}$ \\
\hline Total & 5225 & 48,449 \\
\hline \multicolumn{3}{|l|}{ IBD subtype } \\
\hline $\begin{array}{l}\text { CD } \\
\text { UC } \\
\text { IBD-U }\end{array}$ & $\begin{array}{l}1617(30.9) \\
2893(55.4) \\
715(13.7)\end{array}$ & $\begin{array}{l}13,524(27.9) \\
26,572(54.8) \\
8353(17.2)\end{array}$ \\
\hline \multicolumn{3}{|l|}{ Sex } \\
\hline $\begin{array}{l}\text { Male } \\
\text { Female }\end{array}$ & $\begin{array}{l}2813(55.4) \\
2268(44.6)\end{array}$ & $\begin{array}{l}24,772(51.1) \\
23,677(48.9)\end{array}$ \\
\hline \multicolumn{3}{|c|}{ Age at IBD diagnosis (years) } \\
\hline $\begin{array}{l}\text { Median (IQR) } \\
\text { Mean (SD) } \\
<6 \\
6-<10 \\
10-<18 \\
18-<40 \\
40-<60 \\
\geq 60\end{array}$ & $\begin{array}{l}37(25-53) \\
39(18) \\
35(0.7) \\
96(1.9) \\
501(9.9) \\
2166(42.6) \\
1416(27.9) \\
867(17.1)\end{array}$ & $\begin{array}{l}38(24-58) \\
41(20) \\
239(0.5) \\
502(1.0) \\
4620(9.5) \\
20,246(41.8) \\
12,166(25.1) \\
10,676(22.0)\end{array}$ \\
\hline \multicolumn{3}{|c|}{ Year of IBD diagnosis } \\
\hline $\begin{array}{l}|969-200| \\
2002-2006 \\
2007-201 \mid \\
20 \mid 2-2017\end{array}$ & $\begin{array}{l}1319(25.2) \\
750(14.4) \\
1242(23.8) \\
1914(36.6)\end{array}$ & $\begin{array}{l}0(0.0) \\
15,947(32.9) \\
17,309(35.7) \\
15,193(31.4)\end{array}$ \\
\hline
\end{tabular}

Abbreviations: IBD, inflammatory bowel disease; CD, Crohn's disease; IBD-U, IBD unclassified; UC, ulcerative colitis; NPR, National Patient Register; SWIBREG, Swedish Quality Register for Inflammatory Bowel Disease; ESPRESSO, Epidemiology Strengthened by histoPathology Reports in Sweden; IQR, interquartile range; SD, standard deviation.

(Figure 1). Of 12,155 total SWIBREG assessments, 11,051 (91\%) had a matching histology assessment in ESPRESSO.

\section{Description of the Models Linking SWIBREG and ESPRESSO}

We established rules based on the estimated Bayesian Rule List Model for calculating a histologic inflammation severity score to predict an endoscopic score using the training sample (Algorithm). Briefly, for each individual and location on a given date, 4 scores ranging from 0 to 1 were calculated corresponding to the predicted probability of being in each MES/SCS category. To obtain the predicted class, we took the number with the highest score probability from each class. To obtain the numeric SNOMED-based histology score, we multiplied each probability by the score number $(1,2,3,4)$, took the sum of those, and subtracted one. The score, therefore, represents the expected MES or SCS given the SNOMED histopathology codes. This computation is done for each location (topography code) available at that pathology report date. For use in subsequent analyses, the maximum expected endoscopic score over the available locations of assessment was used for that date.

We then compared the predicted to actual MES/SCS scores using independent data from the validation sample (Table 2) and constructed boxplots of the correspondence between the histology scores from ESPRESSO and endoscopic scores from SWIBREG (Figure 2). We then constructed ROC curves for predicting a nonzero MES/SCS (Figure 3; Table 3). The kappa coefficients for predicted MES/SCS are UC: 0.30, CD: 0.11, IBD-U: 0.28. Overall, the continuous SNOMED-based histology score from ESPRESSO predicted the endoscopic score on unobserved samples with accuracy, but the predicted endoscopic category did not have acceptable performance. To increase interpretability of the rule list, we dichotomized the continuous SNOMED-based histology score into 0 (low histologic inflammation, below cutoff for that IBD subtype) or 1 (high histologic inflammation, above cutoff for that IBD subtype) for use in further analyses. Examining the ROC curves, there was a point at which the curve for the Bayesian rule list score declined rapidly below 90\% true positive fraction (TPF) after tracking the curve for the Lasso model, and thus, we choose the largest cutoff on the histology score where the TPF remained above 0.9. The resulting cutoffs for dichotomizing the SNOMEDbased histology score were UC: $0.77, \mathrm{CD}: 0.68$, IBD-U: 0.64. The clinical score had poor apparent performance and was not pursued further.

\section{IBD-Related Hospitalization in the SWIBREG-ESPRESSO-NPR Linkage}

Among the 2741 patients in the SWIBREG-ESPRESSONPR linkage with an endoscopic assessment within 1 year of their IBD onset, during follow up from the initial IBD diagnosis, 639 patients had a subsequent IBD-related hospitalization. The baseline endoscopic score was associated with time to IBD-related hospitalization, which was strongest for $\mathrm{UC}$, although $\mathrm{CD}$ and IBD-U also reached significance (log-rank UC $\mathrm{p}<0.001, \mathrm{CD} \mathrm{p}=0.020$, IBD-U 

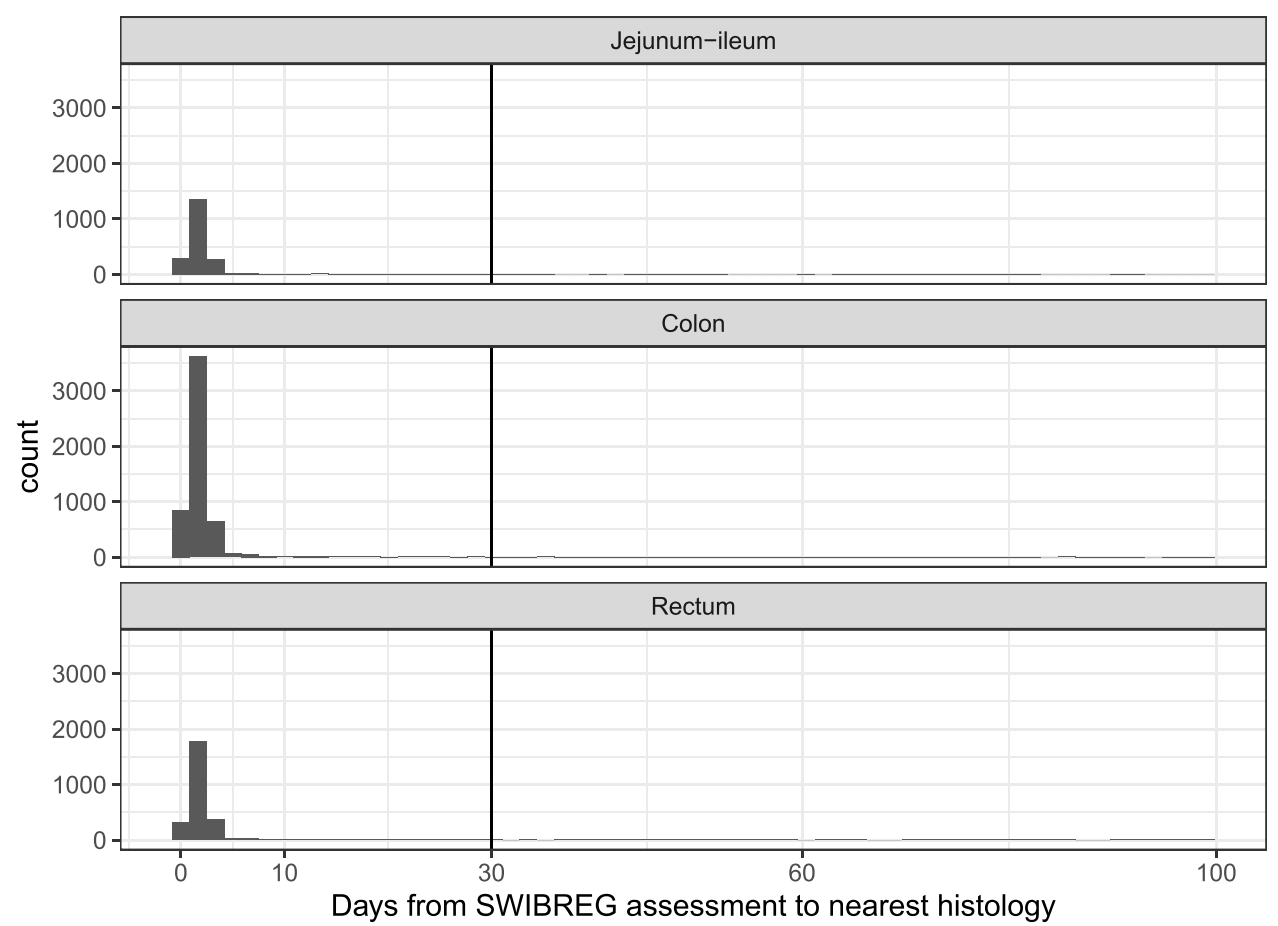

Figure I Temporality of SWIBREG endoscopic assessment linked to a gastrointestinal histopathology specimen in ESPRESSO.

Abbreviations: SWIBREG, Swedish Quality Register for Inflammatory Bowel Disease; ESPRESSO, Epidemiology Strengthened by histoPathology Reports in Sweden.

Table 2 Cross-Tabulation of Predicted Mayo Endoscopic Subscore/SWIBREG-CD Score versus Observed Score. The Predicted Mayo Endoscopic Subscore/SWIBREG-CD Score Rows are Using the Predicted Category as Described in Algorithm

\begin{tabular}{|c|c|c|c|c|}
\hline & Actual MES/SCS: 0 & Actual MES/SCS: I & Actual MES/SCS: 2 & Actual MES/SCS: 3 \\
\hline \multicolumn{5}{|l|}{ UC } \\
\hline Predicted MES: 0 & $682(72.6 \%)$ & 144 (I5.3\%) & $95(10.1 \%)$ & 18 (1.9\%) \\
\hline Predicted MES: I & 43 (24.3\%) & 50 (28.2\%) & $64(36.2 \%)$ & 20 (II.3\%) \\
\hline Predicted MES: 2 & 161 (19.1\%) & 259 (30.7\%) & $326(38.6 \%)$ & 99 (II.7\%) \\
\hline Predicted MES: 3 & $2(50.0 \%)$ & $0(0.0 \%)$ & $0(0.0 \%)$ & $2(50.0 \%)$ \\
\hline High histology score & $336(25.8 \%)$ & $371(28.5 \%)$ & 459 (35.2\%) & 137 (10.5\%) \\
\hline \multicolumn{5}{|l|}{$C D$} \\
\hline Predicted SCS: 0 & $603(55.8 \%)$ & 237 (21.9\%) & $156(14.4 \%)$ & 85 (7.9\%) \\
\hline Predicted SCS: I & $25(25.3 \%)$ & $36(36.4 \%)$ & $19(19.2 \%)$ & 19 (19.2\%) \\
\hline Predicted SCS: 2 & $5(21.7 \%)$ & $9(39.1 \%)$ & $9(39.1 \%)$ & $0(0.0 \%)$ \\
\hline Predicted SCS: 3 & $8(17.4 \%)$ & 15 (32.6\%) & $12(26.1 \%)$ & II (23.9\%) \\
\hline High histology score & $292(35.0 \%)$ & $253(30.3 \%)$ & $179(21.4 \%)$ & $1 \mathrm{II}(13.3 \%)$ \\
\hline \multicolumn{5}{|l|}{ IBD-U } \\
\hline Predicted MES: 0 & $162(71.4 \%)$ & $32(14.1 \%)$ & $26(11.5 \%)$ & $7(3.1 \%)$ \\
\hline Predicted MES: I & - & - & - & - \\
\hline Predicted MES: 2 & 69 (20.6\%) & 99 (29.6\%) & $129(38.5 \%)$ & 38 (1 I.3\%) \\
\hline Predicted MES: 3 & - & - & - & - \\
\hline High histology score & $85 \%(23.0 \%)$ & 107 (28.9\%) & 139 (37.6\%) & $39(10.5 \%)$ \\
\hline
\end{tabular}

Abbreviations: CD, Crohn's disease; IBD-U, IBD unclassified; UC, ulcerative colitis; MES, Mayo endoscopic subscore; SCS, SWIBREG-CD score; SWIBREG, Swedish Quality Register for Inflammatory Bowel Disease. 

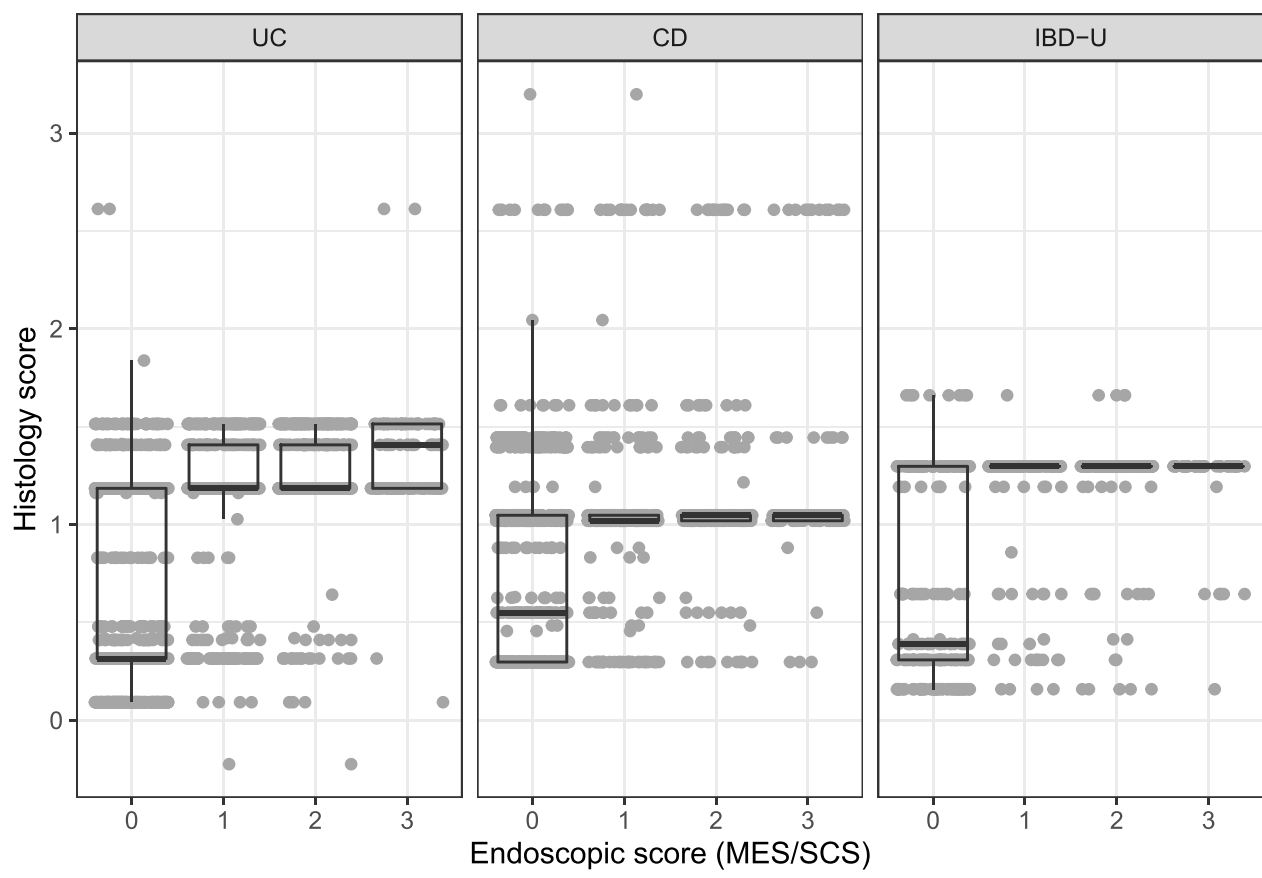

Figure 2 Boxplots of the correspondence between the histology score from ESPRESSO and the endoscopic score from SWIBREG in all patients in the validation subsample $(n=5225)$. P-values from the Kruskal-Wallis rank sum test comparing the histology score by MES/SCS group are $<0.00 \mathrm{I}$ for all IBD subtypes.

Abbreviations: SWIBREG, Swedish Quality Register for Inflammatory Bowel Disease; ESPRESSO, Epidemiology Strengthened by histoPathology Reports in Sweden; CD, Crohn's disease; IBD-U, IBD unclassified; UC, ulcerative colitis; MES, Mayo endoscopic subscore; SCS, SWIBREG-CD score.

$\mathrm{p}<0.001$; Figure 4). The dichotomized baseline SNOMED- inflammation) was associated with time to IBD-related based histology score based on the Bayes model into 0 hospitalization in UC and IBD-U but not CD (log-rank (low histologic inflammation) or 1 (high histologic $\mathrm{UC} \mathrm{p}=0.018, \mathrm{CD} \mathrm{p}=0.960$, IBD-U $\mathrm{p}=0.034$; Figure 5).

\section{ROC curve for nonzero MES/SCS}
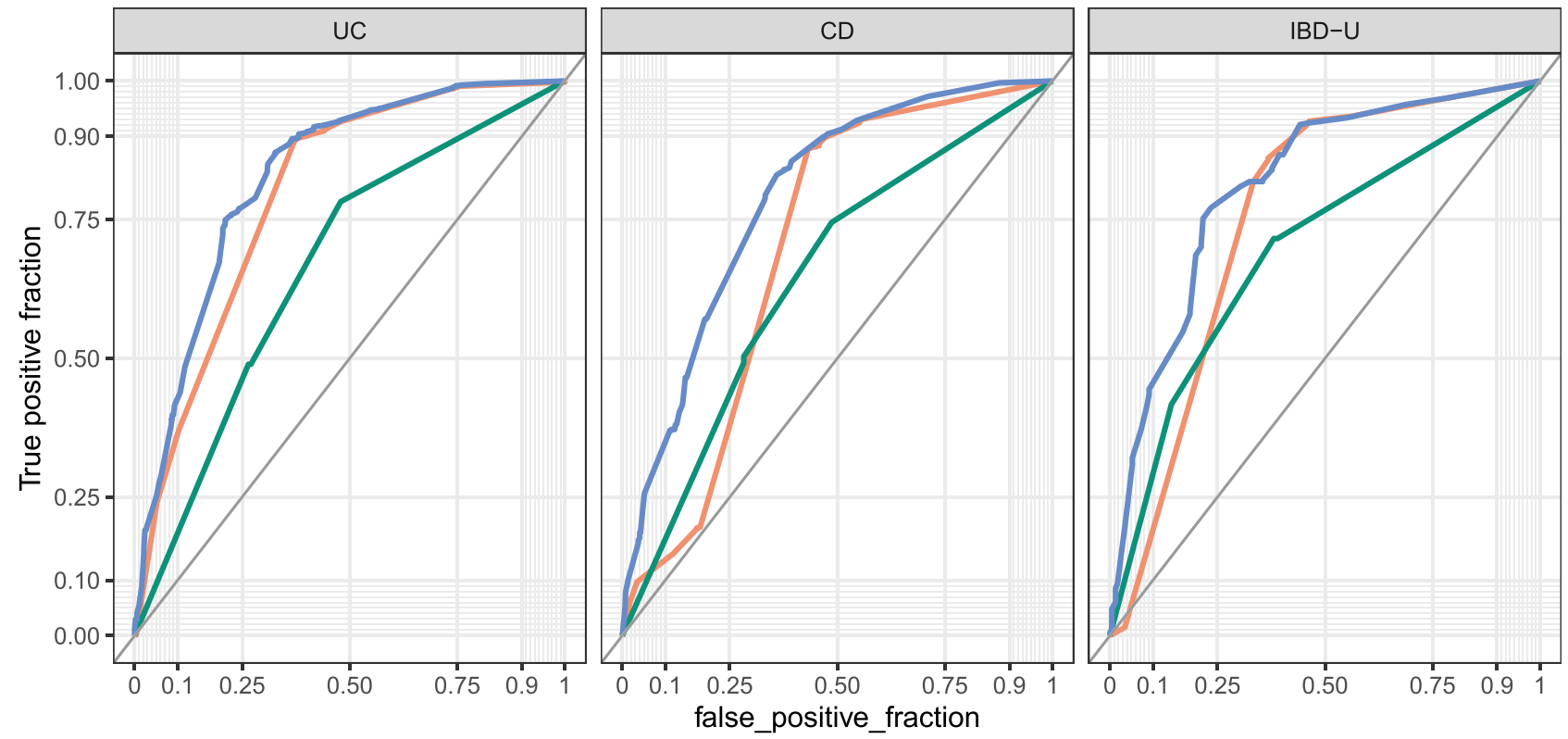

model - Bayes - Clinical - Lasso

Figure 3 ROC curves for nonzero endoscopic score stratified by IBD subtype in the SWIBREG-ESPRESSO-NPR linkage ( $\mathrm{n}=5225$ ).

Abbreviations: ROC, receiver operating characteristic; IBD, inflammatory bowel disease; CD, Crohn's disease; IBD-U, IBD unclassified; UC, ulcerative colitis. 
Table 3 Areas Under the ROC Curves in the Validation Sample by IBD Type and Model with Bootstrap 95\% Confidence Intervals and Permutation Test p-values for Pairwise Comparisons (10,000 Replicates)

\begin{tabular}{|c|c|c|c|c|c|c|}
\hline IBD Subtype & Bayes & Lasso & Clinical & Lasso-Bayes & Bayes-Clinical & Lasso-Clincal \\
\hline & \multicolumn{3}{|c|}{ Area under ROC curve $(95 \% \mathrm{Cl})$} & \multicolumn{3}{|l|}{ P-value } \\
\hline UC & $0.80(0.78-0.81)$ & $0.83(0.82-0.84)$ & $0.66(0.65-0.68)$ & 0.016 & $<0.001$ & $<0.001$ \\
\hline$C D$ & $0.70(0.68-0.72)$ & $0.78(0.77-0.80)$ & $0.65(0.63-0.66)$ & $<0.001$ & 0.0016 & $<0.001$ \\
\hline IBD-U & $0.76(0.73-0.78)$ & $0.82(0.80-0.84)$ & $0.69(0.67-0.72)$ & 0.015 & 0.010 & $<0.001$ \\
\hline
\end{tabular}

Abbreviations: ROC, Receiver operating characteristic; IBD, inflammatory bowel disease; CD, Crohn's disease; IBD-U, IBD unclassified; UC, ulcerative colitis.

In addition, in this subgroup of 2741 patients, we were able to assess the relative importance of the initial endoscopic score and the SNOMED-based histology score in their association with time to IBD-hospitalization. In a multivariable Cox model for time to IBDhospitalization for a dichotomized SNOMED-based histology score adjusted for endoscopic score, sex, and age at IBD onset, a high histology score (aHR 3.18, 95\% CI $1.01-10.03, \mathrm{p}=0.049$ ) was a predictor of IBD-related hospitalization in UC, despite the adjustment for non-zero endoscopic score (aHR 1.66, 95\% CI 0.96-2.86, $\mathrm{p}=0.068$; Table 4). In a multivariable Cox model, adjusting for baseline endoscopic score with 0 as the reference category, sex, and age of IBD-onset, we found insufficient evidence that the continuous SNOMED-based histology score was associated with time to IBD-related hospitalization, though the estimate in UC suggested a $10 \%$ increase in the hazard of IBD-related hospitalization for a unit increase in histology score $(\mathrm{HR}=1.10,95 \%$ CI $(0.70$ to 1.71$)$ ).

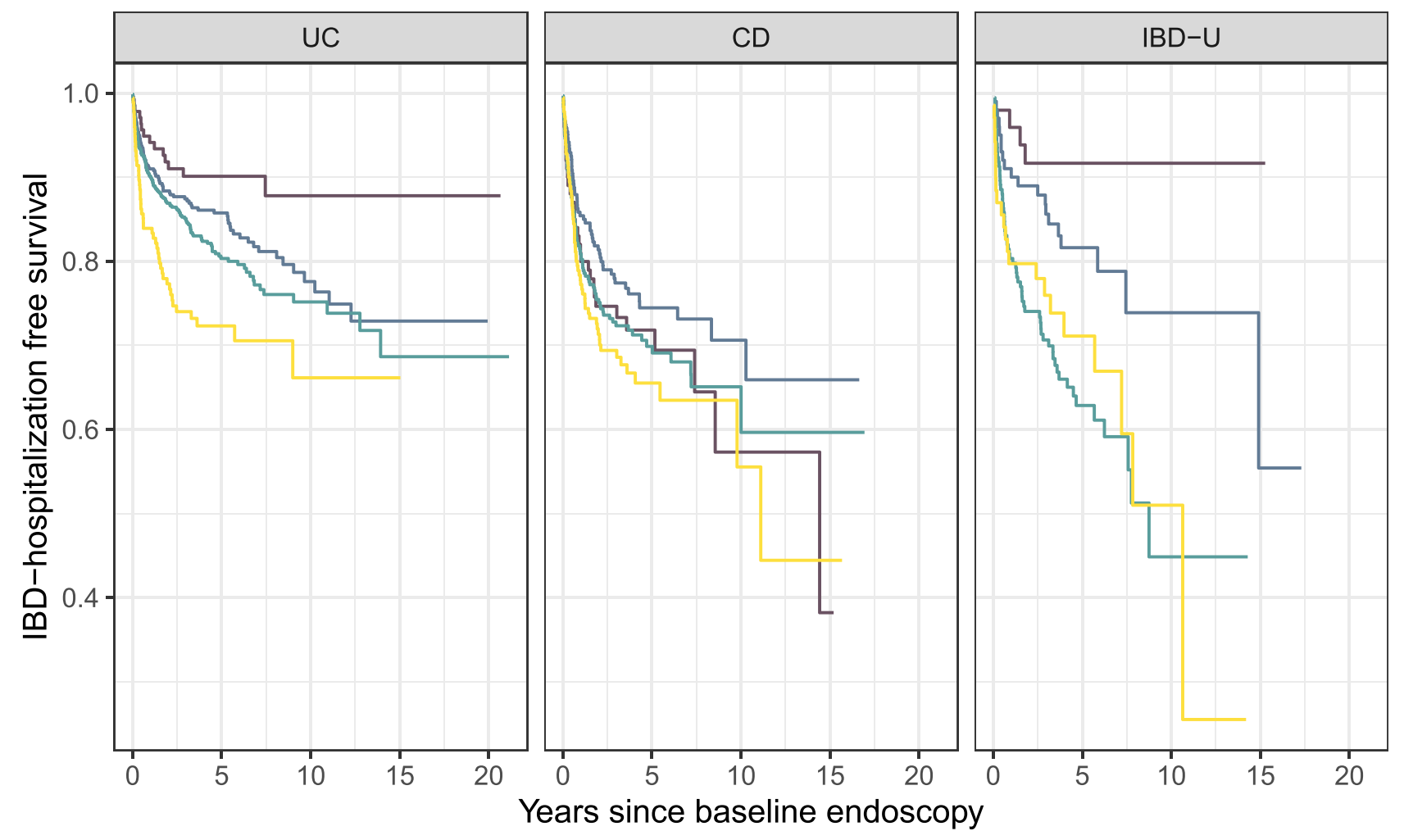

MES/SCS $-0-1-2-3$

Figure 4 Time to IBD-related hospitalization by baseline MES/SCS score in the SWIBREG-ESPRESSO-NPR linkage $(n=5225)$, stratified by IBD subtype. Log-rank $p$-values: UC $p<0.00$ I, CD $p=0.020$, IBD-U $p<0.00$ I.

Abbreviations: IBD, inflammatory bowel disease; CD, Crohn's disease; IBD-U, IBD unclassified; UC, ulcerative colitis; SWIBREG, Swedish Quality Register for Inflammatory Bowel Disease; ESPRESSO, Epidemiology Strengthened by histoPathology Reports in Sweden; MES, Mayo endoscopic subscore; SCS, SWIBREG-CD score. 

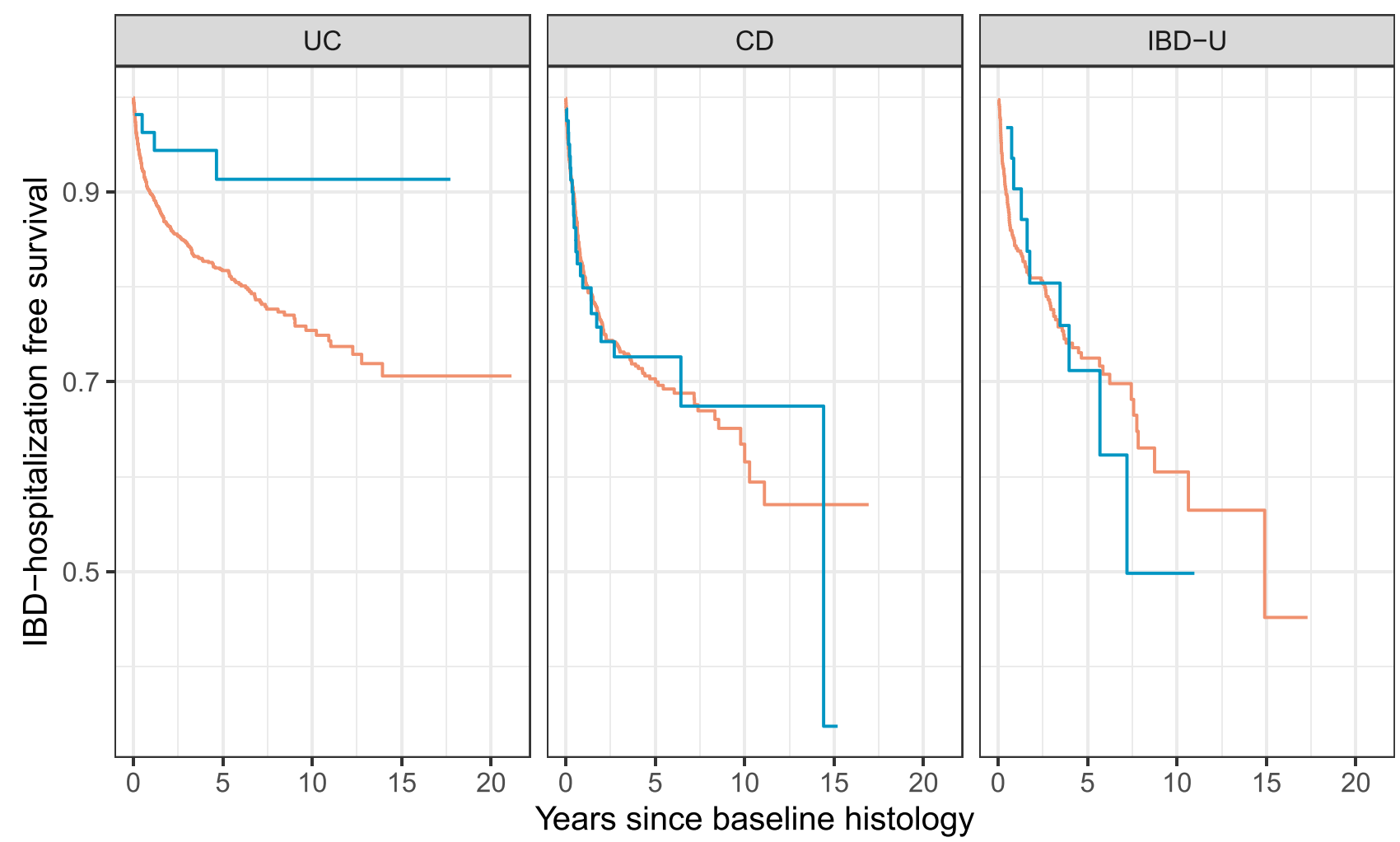

Baseline histology — high — low

Figure 5 Time to IBD-related hospitalization by baseline histology score (Bayes model) in the SWIBREG-ESPRESSO-NPR linkage ( $\mathrm{n}=5225$ ), stratified by IBD subtype. Log rank test $p$-values: $U C p=0.018, C D p=0.960, I B D-U p=0.034$.

Abbreviations: IBD, inflammatory bowel disease; CD, Crohn's disease; IBD-U, IBD unclassified; UC, ulcerative colitis; NPR, National Patient Register; SWIBREG, Swedish Quality Register for Inflammatory Bowel Disease; ESPRESSO, Epidemiology Strengthened by histoPathology Reports in Sweden.

\section{IBD-Related Hospitalization in the ESPRESSO-NPR Linkage}

In the larger sample of 48,449 individuals with an initial IBD diagnosis in the NPR on or after 2002 with 12,134 observed IBD-hospitalization events, using the ESPRESSO SNOMED-based histology score at baseline, we observe similar associations with time to IBD-related hospitalization in this larger sample, with a strong association seen in $\mathrm{UC}$ and $\mathrm{CD}$ patients, but no association in IBD-U patients (log-rank p-value: UC: $<0.001, \mathrm{CD}$ p-value $<0.001$, IBD-U p-value: 0.996; Figure 6). We were able to estimate this association with greater precision using the SNOMED-based histology score due to the larger sample and longer average follow up time. In a Cox model, adjusted for sex, and age of IBD onset, both the continuous and dichotomized SNOMED-based histology scores were strongly associated with time to IBD-related hospitalization in UC patients (Table 5).

\section{Discussion Main Findings}

In this population-based study of incident IBD patients in Sweden, we used a data driven approach to create a histologic score based on SNOMED coding in ESPRESSO that accurately predicted endoscopic scores in SWIBREG. This model was particularly robust for predicting a zero versus a nonzero endoscopic score, consistent with $\mathrm{EH}$, and validating ESPRESSO for the study of IBD. The model is clinically interpretable and easy to communicate as the scores are determined by a simple series of logical rules based on the presence or absence of common pathology codes. The model was also more reliable in $\mathrm{UC}$ than in $\mathrm{CD}$, as complications of $\mathrm{CD}$ may not always be linked to luminal endoscopic or histologic activity. Finally, we clinically validated our SNOMEDbased histology score by demonstrating that both SWIBREG endoscopic scores of endoscopic inflammation 
Table 4 Hazard Ratios and 95\% Confidence Intervals for Time to IBD-Hospitalization in Mutually Adjusted Cox Models for Histology Score and Endoscopic Score in the SWIBREGESPRESSO-NPR Linkage. Models are Also Adjusted for Sex and Age at IBD Onset

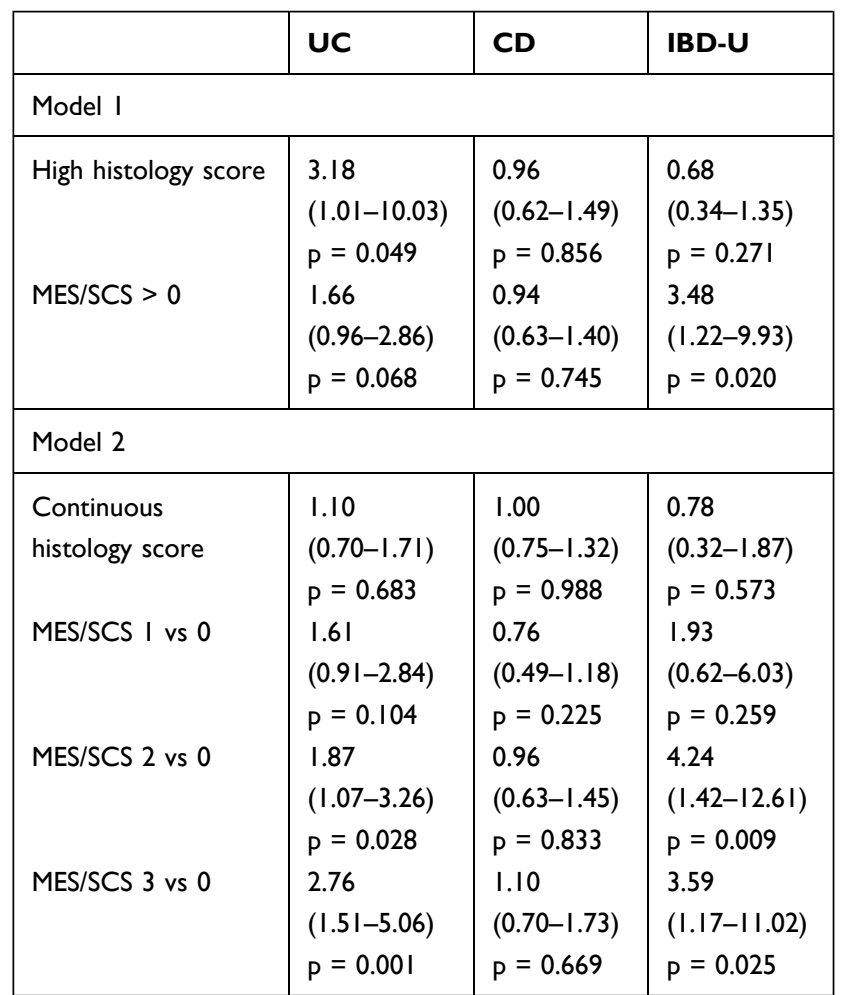

Abbreviations: IBD, inflammatory bowel disease; CD, Crohn's disease; IBD-U, IBD unclassified; UC, ulcerative colitis; NPR, National Patient Register; SWIBREG, Swedish Quality Register for Inflammatory Bowel Disease; ESPRESSO, Epidemiology Strengthened by histoPathology Reports in Sweden; MES, Mayo endoscopic subscore; SCS, SWIBREG-CD score.

and our developed ESPRESSO SNOMED-based histology score were strongly associated with future IBD-related hospitalizations.

\section{Importance of Histology Model}

Based on our predictive model, we have identified several ESPRESSO topography and morphology codes that in combination are highly characteristic of an endoscopic score greater than zero. The SNOMED-based histology score is a highly informative variable that may be applied as a continuous or dichotomized exposure to assess the impact of baseline histology on pertinent outcomes in IBD on a population-level. While we only examined baseline macroscopic endoscopic inflammation severity by MES and SCS in SWIBREG and baseline microscopic histologic inflammation severity by a computed histology score in ESPRESSO, these exposure variables may be measured longitudinally in a large population-based sample, and at many time points. In addition, as many providers may not quantify endoscopic activity using the MES and SCS, the histology score from biopsies obtained may be used to predict endoscopic scores. These variables may be used to assess the impact of endoscopic and histologic activity, particularly changes in endoscopic and histologic activity consistent with mucosal healing, on the natural course of IBD and important IBD outcomes.

\section{Measures of Inflammation and IBD-Related Hospitalization}

We estimated the association of baseline endoscopic and histology scores on time to IBD-related hospitalization, a common complication of IBD. Our data demonstrated that an elevated baseline MES/SCS, and to a larger extent, an elevated baseline SNOMED-based histology score, both as a dichotomous and continuous variable, was strongly associated with time to IBD-related hospitalization in the first year, especially in UC. Our estimation was more precise using histology than the endoscopic scores as we were able to calculate SNOMED-based histology score on a much larger sample and over a longer period of time. There may be several explanations behind the stronger association in UC compared to $\mathrm{CD}$. The connection between endoscopic and histologic findings and future complications seems less strong in patients with $\mathrm{CD},{ }^{46}$ who may require hospitalization for complications of disease independent of luminal endoscopic or histologic inflammatory activity such as for a fibrostenotic (eg, bowel obstruction due to a fibrotic stricture) or penetrating disease (eg, abscess).

Few studies, largely in UC, have examined the correlation between validated endoscopic and histological indices. ${ }^{2,25,47}$ In general, endoscopic scores correlate moderately well with histologic scores, particularly in active disease. In a single-center study of 131 patients with UC, there was modest correlation between the Mayo endoscopic subscore and Riley histologic score (Kendall's $[\mathrm{tau}]=0.482, \mathrm{P}<0.0001) .{ }^{14}$ In another single center study, the ulcerative colitis endoscopic index of severity (UCEIS) strongly correlated with the Nancy ( $\mathrm{r}=$ 0.84, 95\% CI 0.76-0.89, p < 0.001) and Robarts' histological indices ( $r=0.86,95 \%$ CI $0.80-0.90, \mathrm{p}<0.001)$. In contrast, in a multicenter prospective cohort study of 100 adults with UC in clinical remission undergoing surveillance colonoscopy for dysplasia, correlations among endoscopic indices, histological subcomponents, 

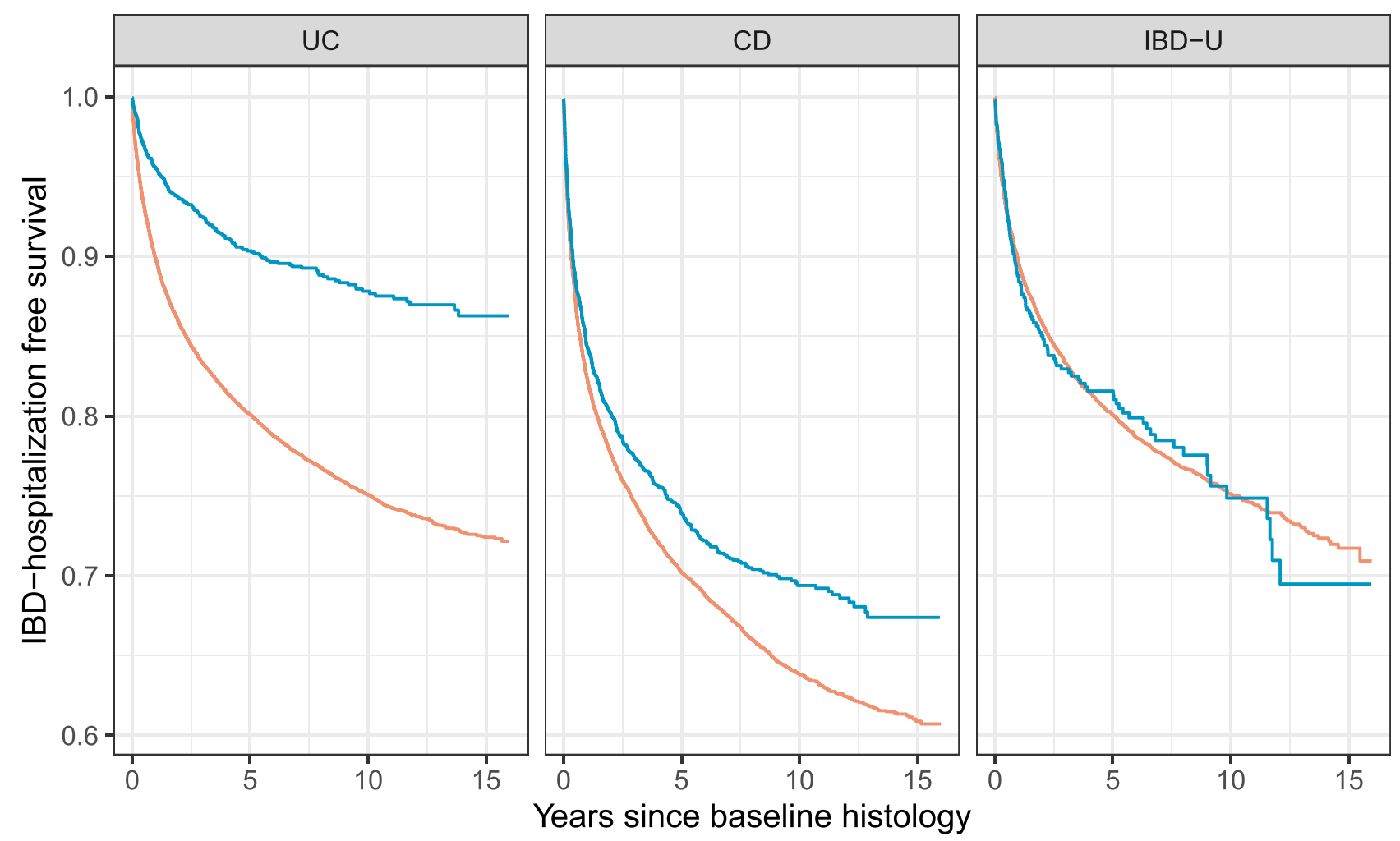

\section{Baseline histology — high — low}

Figure 6 Association between histology score and time to IBD-related hospitalization in an analysis all individuals (ESPRESSO-NPR linkage, $n=48,449)$ with IBD in the histopathology register since 2002. Log-rank p-values: UC: <0.00I, CD p-value <0.00I, IBD-U p-value: 0.996.

Abbreviations: IBD, inflammatory bowel disease; CD, Crohn's disease; IBD-U, IBD unclassified; UC, ulcerative colitis.

and total Riley score were low (total Riley histology score, $\mathrm{r}=0.35-0.37){ }^{25}$ In 101 patients with $\mathrm{CD}$, the level of agreement between endoscopic and histologic activity was fair at $62 \%(\mathrm{~K}=0.2250, \mathrm{P}=0.0064){ }^{22}$

Several studies have demonstrated that $\mathrm{HH}$ is associated with improved clinical outcomes, beyond EH, and

Table 5 Hazard Ratios and 95\% Confidence Intervals for Time to IBD-Hospitalization Adjusted for Sex, and Age of IBD Onset, for a Continuous Histology Score and High versus Low Histology Score

\begin{tabular}{|l|l|l|l|}
\hline & UC & CD & IBD-U \\
\hline Continuous & 1.47 & $I .17$ & 1.04 \\
histology score & $(I .36-1.59)$, & $(I .10-1.25)$, & $(0.85-1.29)$, \\
& $\mathrm{P}<0.00 \mathrm{I}$ & $\mathrm{P}<0.00 \mathrm{I}$ & $\mathrm{P}=0.68 \mathrm{I}$ \\
Dichotomous & 2.26 & $\mathrm{I} .20$ & 1.02 \\
histology score & $(1.93-2.64)$, & $(\mathrm{I} .10-1.3 \mathrm{I})$, & $(0.85-1.24)$, \\
& $\mathrm{P}<0.00 \mathrm{I}$ & $\mathrm{P}<0.00 \mathrm{I}$ & $\mathrm{P}=0.818$ \\
\hline
\end{tabular}

Abbreviations: IBD, inflammatory bowel disease; CD, Crohn's disease; IBD-U, IBD unclassified; UC, ulcerative colitis. is a negative predictor of relapse and of colorectal neoplasia risk..$^{7,12,13,16-23,25,48,49}$ In a study of 646 patients with UC, histologic normalization of colonic mucosa was associated with an increased odds of relapse-free survival compared with EH or histologic quiescence. ${ }^{49}$ While studies of HH have largely focused on UC, in the aforementioned study of 101 patients with ileal CD in clinical remission followed for a median of 21 months, HH but not EH was associated with a decreased risk of clinical relapse, escalation of medical therapy, and corticosteroid use. $^{22}$

In future studies, utilizing clinical and endoscopic data from SWIBREG and histologic data from ESPRESSO, we will be able to quantitatively assess for the impact of healing on critical IBD outcomes, such as surgery and corticosteroid use, on a population-based level. In addition, we will be able to integrate IBD medication exposures to comparatively assess specific classes and durations of medications on mucosal healing. 


\section{Strengths and Limitations}

The main strengths of our study are its population-based large cohort size, prospectively recorded data in routine medical practice, with a long follow-up period, extracted from national registers. As well, the study used validated exposure definitions and had virtually complete capture of outcome measures. The histology score could also be more broadly applied to other sources of histology, such as histology databases in the United States, that are subsequently coded by SNOMED. Alternatively, if suitable data sources are available, similar methodology could be used to develop a scoring system based on electronic records of histologybased diagnoses. The present study also has limitations, including lack of stratification by IBD extent and phenotype and inability to use a validated endoscopic score in CD and histology score, such as the Robarts' Histopathology Index ${ }^{50}$ the Nancy Index, ${ }^{51}$ or the Geboes score ${ }^{52}$ in UC; no such validated histology score exists in $\mathrm{CD}$. Due to the relatively smaller sample of patients with IBD-U, the classification into endoscopic cohorts by the algorithm was limited. Given limitations in morphology coding, while we were able to differentiate the presence or absence of inflammation, we were unable to fully assess for subtleties differentiating severe from mild inflammation. Given the heterogeneity in areas biopsied during endoscopy, we could not fully assess the distribution of histologic disease based on topography coding. Moreover, as patients do not systematically undergo routine endoscopy and biopsy, but rather for specific indications such as an exacerbation in symptoms or failure of medical therapy, there could be bias in the data generated towards a more active IBD population.

\section{Conclusion}

In summary, in this population-based cohort study using Swedish patient registers, we linked clinical data in SWIBREG with histopathology data in ESPRESSO for the study of IBD. Linkage and validation are critical to evaluate IBD activity and prognosis as a function of degree and/or duration of intestinal inflammation. Our computed SNOMED-based histology score from ESPRESSO is a valid measure of intestinal inflammation for the study of IBD. Baseline endoscopic and histologic scores are associated with time to IBD-related hospitalization, particularly in UC.

\section{Abbreviations}

CI, confidence interval; NPR, National Patient Register; SWIBREG, Swedish Quality Register for Inflammatory
Bowel Disease; ESPRESSO, Epidemiology Strengthened by histoPathology Reports in Sweden; IBD, inflammatory bowel disease; $\mathrm{CD}$, Crohn's disease; UC, ulcerative colitis; $\mathrm{EH}$, endoscopic healing; $\mathrm{HH}$, histologic healing; OR, odds ratio; SnoMed, Systematized Nomenclature of Medicine; MES, Mayo endoscopic score; SCS, SWIBREG Crohn's disease score; TPF, true positive fraction.

\section{Acknowledgments}

The SWIBREG Study Group: Michael Eberhardson, Ulrika L Fagerberg, Olof Grip, Jonas Halfvarson, Henrik Hjortswang, Susanna Jäghult, Jonas F Ludvigsson, Pär Myrelid, Caroline Nordenvall, Ola Olén, Martin Rejler, and Hans Strid.

\section{Author Contributions}

All authors contributed to data analysis, drafting or revising the article, have agreed on the journal to which the article will be submitted, gave final approval of the version to be published, and agree to be accountable for all aspects of the work.

\section{Funding}

The work was supported by the Karolinska Institutet. Dr. Olén was supported by grants from the Swedish Medical Society, Karolinska Institutet Foundations, Young Scholar Award from the Strategic Research Area Epidemiology program at Karolinska Institutet, and Regional Agreement on Medical Training and Clinical Research between Stockholm County Council and Karolinska Institutet (ALF). The study sponsors had no role in the study design; data collection; data analysis; and interpretation of data; writing of the report; and the decision to submit the paper for publication.

\section{Disclosure}

Dr. Ludvigsson coordinates a study on behalf of the Swedish IBD quality register (SWIBREG) and that study has received funding from Janssen corporation. Dr. Olén has been PI on projects at Karolinska Institutet partly financed by investigator-initiated grants from Janssen and Ferring, and also reports a grant from Pfizer in the context of a national safety monitoring program. None of those studies have any relation to the present study. Karolinska Institutet also has received fees for Olén's lectures and participation on advisory boards from Janssen, Ferring, Takeda, and Pfizer regarding topics not related to the present study. The authors report no other potential conflicts of interest for this work. 


\section{References}

1. Ungaro R, Mehandru S, Allen PB, et al. Ulcerative colitis. Lancet. 2017;389:1756-1770. doi:10.1016/S0140-6736(16)32126-2

2. Torres J, Mehandru S, Colombel J-F, et al. Crohn's disease. Lancet. 2017;389:1741-1755. doi:10.1016/S0140-6736(16)31711-1

3. Axelrad JE, Lichtiger S, Yajnik V. Inflammatory bowel disease and cancer: the role of inflammation, immunosuppression, and cancer treatment. World J Gastroenterol. 2016;22:4794-4801. doi:10.3748/ wjg.v22.i20.4794

4. Lichtenstein GR, Loftus EV, Isaacs KL, et al. ACG clinical guideline: management of Crohn's disease in adults. Am J Gastroenterol. 2018;113:481-517. doi:10.1038/ajg.2018.27

5. Rubin DT, Ananthakrishnan AN, Siegel CA, et al. ACG clinical guideline: ulcerative colitis in adults. $A m$ J Gastroenterol. 2019;114:384-413. doi:10.14309/ajg.0000000000000152

6. Shah SC, Colombel J-F, Sands BE, et al. Mucosal healing is associated with improved long-term outcomes of patients with ulcerative colitis: a systematic review and meta-analysis. Clin Gastroenterol Hepatol. 2016;14:1245-1255.e8. doi:10.1016/j.cgh.2016.01.015

7. Rutter M, Saunders B, Wilkinson K, et al. Severity of inflammation is a risk factor for colorectal neoplasia in ulcerative colitis. Gastroenterology. 2004;126:451-459. doi:10.1053/j.gastro.2003.11.010

8. Lichtenstein GR, Rutgeerts P. Importance of mucosal healing in ulcerative colitis. Inflamm Bowel Dis. 2010;16:338-346. doi:10.1002/ibd.20997

9. Falvey JD, Hoskin T, Meijer B, et al. Disease activity assessment in IBD: clinical indices and biomarkers fail to predict endoscopic remission. Inflamm Bowel Dis. 2015;21:824-831. doi:10.1097/ MIB.0000000000000341

10. Pineton de Chambrun G, Peyrin-Biroulet L, Lémann M, et al. Clinical implications of mucosal healing for the management of IBD. Nat Rev Gastroenterol Hepatol. 2010;7:15-29. doi:10.1038/ nrgastro.2009.203

11. Rosenberg L, Nanda KS, Zenlea T, et al. Histologic markers of inflammation in patients with ulcerative colitis in clinical remission. Clin Gastroenterol Hepatol. 2013;11:991-996. doi:10.1016/j. cgh.2013.02.030

12. Bryant RV, Burger DC, Delo J, et al. Beyond endoscopic mucosal healing in UC: histological remission better predicts corticosteroid use and hospitalisation over 6 years of follow-up. Gut. 2016;65:408-414. doi:10.1136/gutjnl-2015-309598

13. Bessissow T, Lemmens B, Ferrante M, et al. Prognostic value of serologic and histologic markers on clinical relapse in ulcerative colitis patients with mucosal healing. Am J Gastroenterol. 2012;107:1684-1692. doi:10.1038/ajg.2012.301

14. Lemmens B, Arijs I, Van Assche G, et al. Correlation between the endoscopic and histologic score in assessing the activity of ulcerative colitis. Inflamm Bowel Dis. 2013;19:1194-1201. doi:10.1097/ MIB.0b013e318280e75f

15. Pai RK, Jairath V, Vande Casteele N, et al. The emerging role of histologic disease activity assessment in ulcerative colitis. Gastrointest Endosc. 2018;88:887-898. doi:10.1016/j. gie.2018.08.018

16. Park S, Abdi T, Gentry M, et al. Histological disease activity as a predictor of clinical relapse among patients with ulcerative colitis: systematic review and meta-analysis. $\mathrm{Am} \quad J$ Gastroenterol. 2016;111:1692-1701. doi:10.1038/ajg.2016.418

17. Zenlea T, Yee EU, Rosenberg L, et al. Histology grade is independently associated with relapse risk in patients with ulcerative colitis in clinical remission: A prospective study. Am J Gastroenterol. 2016;111:685-690. doi:10.1038/ajg.2016.50

18. Gupta RB, Harpaz N, Itzkowitz S, et al. Histologic inflammation is a risk factor for progression to colorectal neoplasia in ulcerative colitis: a cohort study. Gastroenterology. 2007;133:1099-105; quiz 1340. doi:10.1053/j.gastro.2007.08.001
19. Calafat M, Lobatón T, Hernández-Gallego A, et al. Acute histological inflammatory activity is associated with clinical relapse in patients with ulcerative colitis in clinical and endoscopic remission. Dig Liver Dis. 2017;49:1327-1331. doi:10.1016/j.dld.2017.08.041

20. Peyrin-Biroulet L, Bressenot A, Kampman W. Histologic remission: the ultimate therapeutic goal in ulcerative colitis? Clin Gastroenterol Hepatol. 2014;12:929-34.e2. doi:10.1016/j.cgh.2013.07.022

21. Frøslie KF, Jahnsen J, Moum BA, et al. Mucosal healing in inflammatory bowel disease: results from a Norwegian population-based cohort. Gastroenterology. 2007;133:412-422. doi:10.1053/j.gastro.2007.05.051

22. Christensen B, Erlich J, Gibson PR, et al. Histologic healing is more strongly associated with clinical outcomes in ileal Crohn's disease than endoscopic healing. Clin Gastroenterol Hepatol. 2019. doi:10.1016/j.cgh.2019.11.056

23. Colman RJ, Rubin DT. Histological inflammation increases the risk of colorectal neoplasia in ulcerative colitis: a systematic review. Intest Res. 2016;14:202-210. doi:10.5217/ir.2016.14.3.202

24. Rubin DT, Huo D, Kinnucan JA, et al. Inflammation is an independent risk factor for colonic neoplasia in patients with ulcerative colitis: a case-control study. Clin Gastroenterol Hepatol. 2013;11:1601-8.e1. doi:10.1016/j.cgh.2013.06.023

25. Osterman MT, Scott FI, Fogt FF, et al. Endoscopic and histological assessment, correlation, and relapse in clinically quiescent ulcerative colitis (MARQUEE). Inflamm Bowel Dis. 2020. doi:10.1093/ibd/ izaa048

26. Olén O, Askling J, Sachs MC, et al. Mortality in adult-onset and elderly-onset IBD: a nationwide register-based cohort study 19642014. Gut. 2019.

27. Olén O, Askling J, Sachs MC, et al. Childhood onset inflammatory bowel disease and risk of cancer: a Swedish nationwide cohort study 19642014. BMJ. 2017;358:j3951. doi:10.1136/bmj.j3951

28. Barlow L, Westergren K, Holmberg L, et al. The completeness of the Swedish cancer register: a sample survey for year 1998. Acta Oncol. 2009;48:27-33. doi:10.1080/02841860802247664

29. Ludvigsson JF, Andersson M, Bengtsson J, et al. Swedish Inflammatory Bowel Disease Register (SWIBREG) - a nationwide quality register. Scand J Gastroenterol. 2019;54:1089-1101. doi:10.1080/00365521.2019.1660799

30. Ludvigsson JF, Lashkariani M. Cohort profile: ESPRESSO (Epidemiology Strengthened by histoPathology Reports in Sweden). Clin Epidemiol. 2019;11:101-114. doi:10.2147/CLEP. S191914

31. Ludvigsson JF, Montgomery SM, Ekbom A, et al. Small-intestinal histopathology and mortality risk in celiac disease. JAMA. 2009;302:1171-1178. doi:10.1001/jama.2009.1320

32. Ludvigsson JF, Brandt L, Montgomery SM. Symptoms and signs in individuals with serology positive for celiac disease but normal mucosa. BMC Gastroenterol. 2009;9:57. doi:10.1186/1471-230X-9-57

33. Bozorg SR, Song M, Emilsson L, et al. Validation of serrated polyps (SPs) in Swedish pathology registers. BMC Gastroenterol. 2019;20:3. doi:10.1186/s12876-019-1134-6

34. Svensson M, Bergman D, Olén O, et al. Validating microscopic colitis (MC) in Swedish pathology registers. Scand J Gastroenterol. 2018;53:1469-1475. doi:10.1080/00365521.2018.1543446

35. Ludvigsson JF, Andersson E, Ekbom A, et al. External review and validation of the Swedish national inpatient register. BMC Public Health. 2011;11:450. doi:10.1186/1471-2458-11-450

36. Ludvigsson JF, Otterblad-Olausson P, Pettersson BU, et al. The Swedish personal identity number: possibilities and pitfalls in healthcare and medical research. Eur J Epidemiol. 2009;24:659-667. doi:10.1007/s10654-009-9350-y

37. Jakobsson GL, Sternegård E, Olén $\mathrm{O}$, et al. Validating inflammatory bowel disease (IBD) in the Swedish National Patient Register and the Swedish Quality Register for IBD (SWIBREG). Scand J Gastroenterol. 2017;52:216-221. doi:10.1080/00365521.2016.1246605 
38. Shrestha S, Olén O, Eriksson C, et al. The use of ICD codes to identify IBD subtypes and phenotypes of the montreal classification in the swedish national patient register. Scand J Gastroenterol. 2020;1-6.

39. Olén O, Erichsen R, Sachs MC, et al. Colorectal cancer in ulcerative colitis: a Scandinavian population-based cohort study. Lancet. 2020;395:123-131. doi:10.1016/S0140-6736(19)32545-0

40. Schroeder KW, Tremaine WJ, Ilstrup DM. Coated oral 5 -aminosalicylic acid therapy for mildly to moderately active ulcerative colitis. Randomized Study N Engl J Med. 1987;317:1625-1629. doi:10.1056/NEJM198712243172603

41. Letham B, Rudin C, McCormick TH, et al. Interpretable classifiers using rules and Bayesian analysis: building a better stroke prediction model. Ann Appl Stat. 2015;9:1350-1371. doi:10.1214/15-AOAS848

42. Friedman J, Hastie T, Tibshirani R. Regularization paths for generalized linear models via coordinate descent. J Stat Softw. 2010;33:1-22. doi:10.18637/jss.v033.i01

43. Yang H, Rudin C, Seltzer M. Scalable bayesian rule lists. arXiv. 2016.

44. Sachs MC. plotROC: A Tool for Plotting ROC Curves. J Stat Softw. 2017;79.

45. Therneau TM. Survival Analysis [R package survival version 3. $1-12]$.

46. Hu AB, Tan W, Deshpande V, et al. Ileal or colonic histologic activity is not associated with clinical relapse in patients with Crohn's disease in endoscopic remission. Clin Gastroenterol Hepatol. 2020. doi: $10.1016 / j . \operatorname{cgh} .2020 .04 .050$
47. Theede K, Holck S, Ibsen P, et al. Level of fecal calprotectin correlates with endoscopic and histologic inflammation and identifies patients with mucosal healing in ulcerative colitis. Clin Gastroenterol Hepatol. 2015;13:1929-36.e1. doi:10.1016/j. cgh.2015.05.038

48. Irani NR, Wang LM, Collins GS, et al. Correlation between endoscopic and histological activity in ulcerative colitis using validated indices. J Crohns Colitis. 2018;12:1151-1157. doi:10.1093/ecco-jcc /jjy081

49. Christensen B, Hanauer SB, Erlich J, et al. Histologic normalization occurs in ulcerative colitis and is associated with improved clinical outcomes. Clin Gastroenterol Hepatol. 2017;15:1557-1564.e1. doi:10.1016/j.cgh.2017.02.016

50. Mosli MH, Feagan BG, Zou G, et al. Development and validation of a histological index for UC. Gut. 2017;66:50-58. doi:10.1136/gutjnl2015-310393

51. Marchal-Bressenot A, Salleron J, Boulagnon-Rombi C, et al. Development and validation of the Nancy histological index for UC. Gut. 2017;66:43-49. doi:10.1136/gutjnl-2015-310187

52. Geboes K, Riddell R, Ost A, et al. A reproducible grading scale for histological assessment of inflammation in ulcerative colitis. Gut. 2000;47:404-409. doi:10.1136/gut.47.3.404
Clinical Epidemiology

\section{Publish your work in this journal}

Clinical Epidemiology is an international, peer-reviewed, open access, online journal focusing on disease and drug epidemiology, identification of risk factors and screening procedures to develop optimal preventative initiatives and programs. Specific topics include: diagnosis, prognosis, treatment, screening, prevention, risk factor modification, systematic reviews, risk \& safety of medical interventions, epidemiology \& biostatistical methods, and evaluation of guidelines, translational medicine, health policies \& economic evaluations. The manuscript management system is completely online and includes a very quick and fair peer-review system, which is all easy to use. 